\title{
Bewertung von diffusen Stoffeinträgen im Rahmen der nationalen Biodiversitätsstrategie
}

\author{
Plädoyer für eine integrierte Umweltbeobachtung
}

\author{
Ulrike Doyle $\cdot$ Christiane Heiß
}

Erhalten: 16. März 2009/Akzeptiert: 29. Juli 2009/Online veröffentlicht: 27. August 2009

(C) Springer-Verlag 2009

Zusammenfassung Hintergrund und Ziel 2007 hat die Bundesregierung eine nationale Strategie zur biologischen Vielfalt verabschiedet. Die Biodiversitätsstrategie fordert die Integration ihrer Qualitäts- und Handlungsziele in andere politische Sektorstrategien. Flächendeckende diffuse Stoffeinträge tragen erheblich zum Verlust der biologischen Vielfalt bei. Ihre Reduktion auf ein langfristig verträgliches Niveau stellt eines der Handlungsziele dar. Der Beitrag soll einen Überblick zu den inhaltlichen und organisatorischen Voraussetzungen für die Umsetzung dieses Ziels geben und wichtige Defizite aufzeigen.

Schwerpunkte Hierzu werden die Umweltbeobachtung und ihre Umsetzung in Deutschland im Zusammenhang mit den Zielen der nationalen Strategie zur biologischen Vielfalt dargestellt. Die Möglichkeiten der Erfolgskontrolle der deutschen und europäischen Biodiversitätsstrategien werden anhand der vollzugsspezifischen Umweltbeobachtung sowie internationaler stoffbezogener Indikatoren behandelt.

Ergebnisse Die Umweltbeobachtung in Deutschland und Europa ist durch eine Vielzahl an Messnetzen gekennzeichnet, die nach Umweltmedien und administrativen Zuständigkeiten getrennt voneinander betrieben werden. Nur ein kleiner Teil der in ihnen erhobenen Daten wird übergreifend über die Messnetz- und Umweltmedien ausgewertet,

Dieser Artikel gibt die persönliche, fachliche Einschätzung der beiden Autorinnen wieder.

Ulrike Doyle

Sachverständigenrat für Umweltfragen (SRU),

Reichpietschufer 60, 10785 Berlin, Deutschland

E-Mail: ulrike.doyle@uba.de

Christiane Heiß $(\square)$

Umweltbundesamt,

Postfach 1406, 06844 Dessau-Roßlau, Deutschland

E-Mail: christiane.heiss@uba.de obwohl die technischen Voraussetzungen für die Datenverknüpfung vorhanden sind.

Diskussion Insbesondere fehlen harmonisierte Mindestanforderungen für eine Erfolgskontrolle der stoffbezogenen Teilziele. Ohne geeignete fachliche und organisatorische Vorgaben und Messnetze bleibt das Ziel, flächendeckende diffuse Stoffeinträge zu reduzieren, nicht überprüfbar. Sowohl Umweltdaten als auch Indikatoren sind für die Erfolgskontrolle der fach- und sektorübergreifenden Biodiversitätsstrategie unabdingbar. Auf Bundes- wie auf Länderebene liegen geeignete Konzepte vor, allerdings fehlt es an politischem Willen, diese auch umzusetzen.

Empfehlungen und Perspektiven Die nationale Biodiversitätsstrategie sollte der Diskussion zu einer integrierten Umweltbeobachtung auf Bundesebene neue Impulse verleihen. Erforderlich wäre eine ressortübergreifende Kooperation, um sowohl die internationale Indikatorendiskussion zu reflektieren als auch die institutionellen Hürden zu ermitteln und Lösungsvorschläge im Hinblick auf die geforderten Beiträge zu entwickeln.

Schlüsselwörter Biologische Vielfalt · Biodiversität · Chemikalien $\cdot$ Monitoring $\cdot$ Nährstoffe .

Umweltbeobachtung

\section{Ecological valuation of diffuse immission of chemicals within the national biodiversity strategy}

\begin{abstract}
Background, aim and scope In 2007, the German government approved a national strategy on biological diversity. The strategy requires the integration of its quality targets and action targets into other policies. In addition to other pressure factors, area-wide diffuse substance discharges contribute considerably to the loss of biological diversity.
\end{abstract}


Reducing these diffuse substance discharges to long-term ecologically compatible levels is one of the main action targets. This paper aims to provide an overview of preconditions for implementing this objective in terms of content as well as organization and to point out important deficits.

Main focus To achieve this, the paper will discuss environmental monitoring and its implementation in Germany drawing on the targets of the national strategy on biological diversity. It will discuss the opportunities for the evaluation of the German and European biodiversity strategies using monitoring required in the execution of environmental laws and regulations as well as international substance-related indicators.

Results Environmental monitoring in Germany and in Europe is characterized by a plurality of monitoring networks operated under different administrative competences and environmental sectors. Only a small part of the collected data is evaluated in a cross-media and cross-sectoral perspective even though the linking of the different data sets is technically possible.

Discussion A key problem is that harmonized minimum requirements to monitor the reduction of diffuse substance discharges are missing. Without the implementation of a cooperative and harmonized monitoring approach and organizational guidelines, the reduction of diffuse substance discharges will not be verifiable. Environmental data as well as indicators are essential to evaluate the success of the national biodiversity strategy. Suitable monitoring concepts are available in the German Federal Republic and its Federal States, nevertheless, a political impulse for their implementation is missing.

Recommendations and perspectives The national biodiversity strategy may reopen the discussion on the necessity of an integrated monitoring. A coordinated approach that refers both to the international indicator-discussion and to institutional obstacles and aims to develop a proposal for integrated monitoring data is necessary.

Keywords Biological diversity · Biodiversity · Chemicals $\cdot$ Monitoring $\cdot$ Nutrients $\cdot$ Environmental monitoring

\section{Hintergrund und Ziel}

Die Umweltbeobachtung stellt im Idealfall entscheidungsrelevante Informationen zum Zustand der Schutzgüter und zu den Entwicklungstrends kritischer Belastungsfaktoren bereit. Auf dieser Datenbasis ermöglicht sie es, Handlungsbedarf differenziert zu erkennen sowie den Erfolg von Schutzstrategien zu bewerten. Diese Anforderung gilt umso mehr, wenn Schutzstrategien durch eine Vielzahl unterschiedlicher Akteure umzusetzen sind und die Erfolge vom
Zusammenwirken unterschiedlicher Maßnahmen abhängen. Schutzziele und -strategien im Umwelt- und Naturschutz werden zunehmend europaweit vereinbart. Die Umsetzung und Erfolgskontrolle obliegt den EU-Mitgliedsstaaten. Diese Anforderungen treffen in der Praxis auf eine Vielfalt etablierter Beobachtungsprogramme, deren Methoden und Routinen nicht harmonisiert sind. Zudem werden die Daten der Umweltbeobachtung in der Regel ohne hinreichende Metadatendokumentation archiviert und lassen sich kaum messprogrammübergreifend zusammenführen (Feretti 2001; Schröder und Schmidt 2003). Dies steht einer integrierenden Auswertung mit einzelnen Ausnahmen (Broecker et al. 2007) entgegen, obwohl schon Ellenberg et al. (1978) ein fachlich fundiertes und sachlogisch stringentes Konzept für ein auf Umwelt- und Entwicklungsplanung orientiertes Umweltinformationssystem vorgelegt hatten. Dieses Konzept wurde in Forschungsvorhaben des Bundes erprobt und in dem Sondergutachten ,Allgemeine ökologische Umweltbeobachtung“" (SRU 1991) im Hinblick auf die Einführung einer nationalen, integrierenden und sektorübergreifenden Umweltbeobachtung diskutiert. Die Daten der integrierenden Umweltbeobachtung sollten die Möglichkeit eröffnen, die Schwächen der vorwiegend emissionsorientierten Umweltschutzpraxis zu beheben und Umweltschutz stärker wirkungsorientiert, das heißt - wie zum Beispiel im Biomonitoring (Genßler et al. 2001) - am Schutzgut orientiert, auszurichten. Schwerpunkt sollte ein sogenanntes Ökomonitoring sein, mit dessen Hilfe schleichende oder additive Wirkungen, z. B. von Chemikalien oder der Flächennutzung auf Arten, Artengemeinschaften und Ökosysteme frühzeitig nachzuweisen seien.

Seither erarbeiteten das Bundesamt für Naturschutz und das Umweltbundesamt verschiedene Konzepte für eine nationale Umweltbeobachtung, um dem wachsenden Bedarf an schutzgutübergreifenden Informationen für die internationalen Schutzstrategien nachzukommen (UBA 2002). Die Kernfrage hierbei lautete, wie die vorhandenen Umweltbeobachtungsprogramme und -routinen möglichst effizient an die sich verändernden Anforderungen angepasst werden können. Einzelne Länder und auch der Bund setzten modellhaft integrierende Beobachtungsprogramme um (z.B. für das Biosphärenreservat Rhön: Schönthaler und von Andrian-Wehrburg 2009).

Die Novellierung des BNatSchG im Jahr 2002 führte die Umweltbeobachtung als Aufgabe von Bund und Ländern neu in das Bundesnaturschutzrecht ein ( $\$ 12$ BNatSchG) und verankerte sie auf Bundesebene. Unter Umweltbeobachtung ist nach $\S 12$ BNatSchG ein umfassendes Monitoring $\mathrm{zu}$ verstehen, das auch eine Erfolgskontrolle der medialen Umweltgesetze einschließt (SRU 2002, Tz. 2). Umweltbeobachtung umfasst nach diesem Verständnis sowohl die Struktur von Ökosystemen als auch deren funktionale Aspekte. 
Parallel zu dieser nationalen Reformdiskussion entwickelte die Europäische Kommission umfassende Vorgaben zur Auswertung von Umweltdaten und zur Erfolgskontrolle ihrer Schutzstrategien, sodass die zunächst fachlich motivierten Forderungen nach einer integrierten Umweltbeobachtung und -bewertung mittlerweile von den Informationsanforderungen der EU-Gesetzgebung dominiert werden. Auf europäischer Ebene erfordern u. a. die Umsetzung der Fauna-Flora-Habitat-Richtlinie (FFH-RL, 92/43/EWG), die Vogelschutzrichtlinie (79/409/EWG) sowie das Abkommen zur Erhaltung der Fledermäuse in Europa (EUROBATS) ein Naturschutzmonitoring im engeren Sinne (Dröschmeister et al. 2006).

Die Umsetzung der medialen Schutzstrategien (Wasserrahmen-Richtlinie (2000/60/EG), IVU-Richtlinie (2008/1/ EG) u.w.) erfordern sowohl die Erfassung von Schadstoffquellen als auch von Immissionsbelastungen, um vordringlichen Handlungsbedarf zu bestimmen und die Maßnahmen effektiv zu gestalten. Beispiel hierfür ist das Pollutant Release and Transfer Register (PRTR; BMU 2006), das Unternehmen zu Berichten über 91 Schadstoffe verpflichtet (EG/166/2006).

Eine flächendeckende Bewertung von Schadstoffbelastungen terrestrischer Ökosysteme erfolgt erst für eine sehr begrenzte Stoffanzahl, z. B. für Schwermetalle und Stickstoff auf Grundlage des Moosmonitorings (Harmens et al. 2008, 2009), bodennahes Ozon und versauernde Luftschadstoffe auf Basis der Genfer Luftreinhaltekonvention (UNECE 1979). Die OSPAR-Vertragsstaaten beobachten 42 persistente, bioakkumulierende und toxische (PBT-)Stoffe im Nordostatlantik, um die Maßnahmen zur Reduzierung der Stoffbelastungen bis zum natürlichen Hintergrundniveau zu überwachen (OSPAR 2007). Diese Programme ermöglichen eine wissenschaftlich fundierte Maßnahmenplanung, die den lokalen Bedingungen Rechnung trägt, ohne den Informationsbedarf für die internationale Erfolgskontrolle zu vernachlässigen.

\section{Schwerpunkte}

\subsection{Umweltbeobachtung und deren Umsetzung in Deutschland}

Obwohl die wissenschaftliche und politische Notwendigkeit einer integrierten Umweltbeobachtung seit Anfang der 1990er Jahre zwischen Bund und Ländern Konsens ist, steht die Umsetzung nach wie vor aus (siehe Kap. 1). Insofern gelten die Kernaussagen des Sachstandsberichts (Knetsch und Rosenkranz 2003) sowie die Empfehlungen des SRU (SRU 2004, Kap. 3.3) unverändert: Die Defizite in der Umweltbeobachtung liegen vor allem in der Verknüpfung der vorhandenen Programme untereinander und ihrer inte- grierten Auswertung für die geänderte Informationsnachfrage. Seit Dezember 1998 wurden Konzepte zur Reform der Umweltbeobachtung intensiv zwischen Bund und Ländern diskutiert (BMU 2000; LANA 2003). Demgegenüber berücksichtigen Bund und Länder im Rahmen ihrer Arbeiten zur nachhaltigen Entwicklung vorrangig die Entwicklung gemeinsamer Indikatoren. Zur 70. Umweltministerkonferenz wurde der zweite Erfahrungsbericht (Indikatoren der Bund-Länder-AG Nachhaltige Entwicklung) vorgelegt (UMK 2007). Die Umweltministerkonferenz stimmte auch der Einrichtung eines ständigen Ausschusses „Umweltinformationssysteme" (UIS) innerhalb der Bund/Länder-Arbeitsgemeinschaft „Klima, Energie, Mobilität - Nachhaltigkeit“ (BLAG KliNa) für die IT-Koordinierung der Daten zu.

Die Weiterentwicklung der Umweltbeobachtung erfordert eine unterstützende Rechtsetzung auf Bundesebene. Der Gesetzentwurf zum BNatSchG 2009 (ehemals Entwurf zum UGB, Drittes Buch, nachfolgend BNatSchG-E 2009) konterkariert die bisherigen Entwicklungen auf internationaler Ebene und die konzeptionellen Vorarbeiten auf Bundes- und Länderebene. Der BNatSchG-E 2009 reduziert Umweltbeobachtung ( $\$ 12$ BNatSchG, 2002) auf die nicht näher bestimmte Beobachtung von „Natur und Landschaft“ ( $\$ 6$ BNatSchG-E) und nimmt den integrierenden Ansatz eines umfänglich definierten "Naturhaushaltes“ in $\S 12$ BNatSchG wieder zurück (vgl. Koch und Krohn 2008). Damit bleibt der Ansatz des Übereinkommens über die biologische Vielfalt (Art. 1), Schutz und Nutzung zu integrieren (Abschn. 2.2), in der Umweltbeobachtung und der Erfolgskontrolle unberücksichtigt. In $\S 6$ Abs. 5 BNatSchG-E heißt es zudem: „Das Bundesamt für Naturschutz nimmt die Aufgaben des Bundes auf dem Gebiet der Beobachtung von Natur und Landschaft wahr, soweit in Rechtsvorschriften nichts anderes bestimmt ist". In der Begründung des Referentenentwurfs zu $\S 6$ wird dazu ausgeführt: „Andere Bundesbehörden, denen nicht nach anderen Rechtsvorschriften Aufgaben aus dem Bereich der Beobachtung von Natur und Landschaft obliegen, sind also nicht zur Beobachtung verpflichtet". Diese Zuweisung blendet auch institutionell die Überschneidungen zwischen dem Schutz der Natur und ihrer Nutzung aus und erschwert die dringend notwendige Kooperation bei der Erfolgskontrolle.

Für die Umsetzung der nationalen Biodiversitätsstrategie bleiben geeignete Daten zur Beschreibung des Umweltzustands und zu dessen Bewertung ein kritischer Punkt, wie nachstehende Stellungnahme des sächsischen Staatsministerium für Umwelt und Landwirtschaft beispielhaft zeigt: „Aus fachlicher Sicht besteht die Sorge, dass viele der Zielwerte nicht erreicht werden können, weil a) Umweltdaten und -beobachtungssysteme, die zur Überwachung der Zielerreichung notwendig sind, vielfach nicht vorhanden sind und mit erheblichen Kosten erhoben bzw. eingerichtet werden müssen“ (Sächsischer Landtag 2008). 
2.2 Eine neue Herausforderung:

Die nationale Strategie zur biologischen Vielfalt

Im Jahr 2007 beschloss die Bundesregierung eine nationale Strategie zur biologischen Vielfalt (BMU 2007), zu deren Verabschiedung sich Deutschland durch die Ratifizierung des Übereinkommens über die biologische Vielfalt (Convention on Biological Diversity, CBD) verpflichtet hat (gemäß Art. 6 CBD). Das Übereinkommen über die biologische Vielfalt wurde 1992 verabschiedet und ist der erste völkerrechtliche Vertrag, der den Umgang mit der Natur umfassend regelt. In den einzelnen Artikeln werden Prinzi- pien und allgemeine Handlungsziele zur Erreichung der drei Hauptziele des Übereinkommens (Art. 1 CBD) genannt:

- Erhaltung der biologischen Vielfalt,

- nachhaltige Nutzung ihrer Bestandteile sowie

- ausgewogene und gerechte Aufteilung der sich aus der Nutzung der genetischen Ressourcen ergebenden Vorteile.

Der Ansatz der CBD geht über die traditionelle Naturschutzpolitik hinaus und verbindet den Habitat- und Artenschutz mit Vorgaben der nachhaltigen Nutzung sowie mit dem sogenannten Vorteilausgleich, der festlegt, dass Nutzer der Biodiversität dafür Gegenwerte liefern. Wir sind für
Abb. 1 Nationale Strategie zur biologischen Vielfalt

\section{Nationale Strategie zur biologischen Vielfalt}

Kapitel: B 3.1 Flächendeckende diffuse Stoffeinträge

Begründungen: Viele Tier- und Pflanzenarten in Deutschland sind durch den Eintrag ferntransportierter Luftschadstoffe (Stickstoffverbindungen, Schwefeloxide, Schwermetalle, POP’s etc.) gefährdet. Zum Schutz empfindlicher Ökosysteme wurden in den UNECE-Luftreinhalte-Protokollen nationale Emissionshöchstmengen und Emissionsminderungsmaßnahmen für Luftschadstoffe vereinbart, die auf ökosystemspezifischen, wirkungsbasierten Schwellenwerten (critical loads und critical levels) basieren. Darüber hinaus sind in der Richtlinie 2001/81/EG (NEC-RL) sowie in der 33. BImSchV Emissionshöchstmengen für vier „klassische“ Luftschadstoffe festgelegt worden. Zur Erhaltung der biologischen Vielfalt der Oberflächengewässer enthält die EG-WRRL die verbindliche Vorgabe eines „guten chemischen und guten ökologischen Zustand“ und für Grundwasser eines „guten chemischen und mengenmäßigen Zustandes“. Für die Meere sind im Rahmen von HELCOM und OSPAR Ziele und Handlungsstrategien zu gefährlichen (PBT-) Stoffen und hinsichtlich der Eutrophierung formuliert worden. Darüber hinaus gibt es für bestimmte Industrieanlagen Emissions- und Einleitungsgrenzwerte. Die Nordseeschutz-Konferenzen haben Reduktionsziele von 50 \% (teilweise auch $70 \%$ ) für Einträge von gefährlichen Stoffen und Nährstoffen in die Nordsee auf der Basis des Jahres 1985 beschlossen. Vergleichbare Beschlüsse haben auch OSPAR für den Nordostatlantik und HELCOM für die Ostsee gefasst. Regelungen zur Vermeidung und Verringerung von POP's sind im Stockholmer POP-Übereinkommen sowie im POP-Protokoll der UNECE enthalten. (...)

\section{Wir streben folgendes an:}

- $\quad$ Fortentwicklung von internationalen Übereinkommen und EU-Regularien (z.B. EG-WRRL, UNECE Longrange Transboundary Air Pollution (LRTAP)-Luftreinhalteprotokolle)

- Festlegung von ökosystembezogenen Wirkungsschwellenwerten für Schadstoffe, die die Auswirkungen auf die biologische Vielfalt beschreiben, bis 2015

- Reduzierung der Schadstoffeinträge in die Meeresumwelt bis 2020 auf das Niveau der natürlichen Hintergrundkonzentrationen und bei synthetischen Stoffen auf nahe Null (HELCOM, OSPAR)

- Entwicklung von Bewertungsmethoden und Qualitätszielen zur Einbeziehung der Grundwasserökologie in den guten Grundwasserzustand bis 2010

- Signifikante Reduktion des Eintrags von Pflanzenschutzmitteln in Böden und Gewässer bis 2015

- Verringerung des Stickstoffüberschusses in der Gesamtbilanz bis 2010 auf $80 \mathrm{~kg} / \mathrm{ha}$, angestrebt wird eine weitere Verringerung bis 2015

- Verminderung des Umwelteintrags von Arzneimitteln, hormonell wirksamen Substanzen und anderen Xenobiotika

- Reduzierung von Schadstoffeinträgen in Böden über alle Eintragspfade auf ein so niedriges Maß, so dass es zu keiner zusätzlichen Schadstoffanreicherung in Böden kommt.

Quelle: BMU 2007, S. 54, 55 
unsere Ernährung, Kleidung und medizinische Versorgung auf eine reiche biologische Vielfalt angewiesen. Die biologische Vielfalt garantiert u. a. sauberes Trinkwasser, fruchtbare Böden, die Bestäubung unserer Kulturpflanzen und die natürliche Schädlingsbekämpfung in der Land- und Forstwirtschaft (sog. ecosystem services).

Der Erfolg der Biodiversitätsstrategie wird wesentlich davon abhängen, wie konkret ihre Ziele in die Sektorstrategien anderer Politikbereiche, in die Gesetzgebung des Bundes sowie in die Umsetzungsbemühungen auf Landesebene eingehen (SRU 2008, Kap. 5.5; Doyle et al. 2005). Die Bundesregierung betrachtet die Biodiversitätsstrategie als ein gesamtgesellschaftliches Programm (BMU 2007, S. 8), an dem daher viele unterschiedliche Akteure mitwirken müssen und das Interessenskonflikte zu bewältigen hat, um vorhandene Nutzungsroutinen zu ändern.

Das Kap. B 3.1 der nationalen Strategie „Umwelteinflüsse auf die biologische Vielfalt" bestimmt, ,flächendeckende diffuse Stoffeinträge" als negativen Faktor (Pressure) für die Erhaltungsziele der Biodiversitätsstrategie. Für einen nachhaltigen Schutz der Biodiversität sollen die genannten stofflichen Einträge auf ein ökologisch verträgliches Maß reduziert werden. Dazu sollen für Schadstoffe ,„̈kosystembezogene Wirkungsschwellenwerte, die die Auswirkungen auf die biologische Vielfalt beschreiben bis 2015" festgelegt werden (Abb. 1).

Die EG schuf rechtliche Grundlagen zur prospektiven Bewertung von Industriechemikalien (REACH-Verordnung (1907/2006/EG), Pflanzenschutzmitteln (91/414/EG), Bioziden (98/8/EG) und zur Umweltbewertung von Humanund Tierarzneimitteln (2001/82/EG; 2001/83/EG)), die die Industrie und die Mitgliedstaaten verpflichten, die Wissenslücken zu den Eintragspfaden und den ökosystem- sowie den gesundheitsbezogenen Wirkungsschwellenwerten bis 2018 zu schließen. Zu den relevanten stofflichen Einträgen zählen auch Verbindungen, die bereits in geringen Konzentrationen dauerhafte Schäden bewirken können. Insbesondere Stoffe, bei denen persistente, bioakkumulierende und toxische Eigenschaften in Kombination vorliegen (sog. PBT-Stoffe), sollten vorsorglich nicht in die Umwelt gelangen. Bestehende Einträge müssen soweit reduziert werden, dass die Belastung natürlichen Hintergrundwerten entspricht. Allerdings fehlen verbindliche Verfahren, wie die Risikoinformationen in die bestehenden Schutz- und Entwicklungsstrategien zu integrieren sind.

\subsection{Erfolgskontrolle auf EU-Ebene:}

Internationale stoffbezogene Indikatoren

Die Europäische Kommission verfolgt und bewertet den Fortschritt ihrer Schutzstrategien mit hochaggregierten Indikatoren im Rahmen des sogenannten DPSIR-Ansatzes. Daten der Umweltbeobachtung fließen als Informationen zum Zustand (State) und den Effekten (Impact) in die Indikatoren ein. Neben den schutzgutbezogenen Daten erfasst das Indikatorsystem auch Informationen zu den gesellschaftlichen Verursachern (Driving forces), zu den Belastungen (Pressure) und zu den Wirkungen der politischen Steuerungsstrategien (Response). Den DPSIR-Ansatz entwickelte die OECD 1989, und er stellt den internationalen Bewertungsrahmen für Umweltpolitik dar. Innerhalb dieses Indikatorensystems erarbeitet und pflegt EUROSTAT die Indikatoren für Response, Driving Forces und Pressure während die State- und Impact-Indikatoren hauptsächlich der Europäischen Umweltagentur (EEA) unterstehen (EUROSTAT 1999, S. 5).

Für die europäische Biodiversitätsstrategie entwickelte die EEA 26 spezifische Indikatoren im Rahmen des Programms SEBI 2010 (Streamlining European 2010 Biodiversity Indicators) (EEA 2007). Das vorgeschlagene Indikatorenset ermöglicht es, die Wirkungen verschiedener Sektorpolitiken auf die Biodiversität pauschal einzuschätzen und nutzt seinerseits bestehende Indikatoren aus anderen Politikstrategien wie z. B. den Indikator Stickstoffüberschuss aus EMEP (European Monitoring and Evaluation Programme) (EEA 2007, S. 89). Ein erster auf diesen Indikatoren beruhender Mid-Term-Bericht zum Zustand der Biodiversität zeigt deren praktische Anwendung und Aussagekraft (Europäische Kommission 2008). Das Indikatorenset enthält lediglich vier Indikatoren mit Nährstoffbezug (Stickstoff, Phosphat und Derivate) (EEA 2007).

Zehn für den Schutz der Biodiversität relevante Stoffindikatoren (Pressure) erfasst EUROSTAT (1999) im Rahmen der Evaluierung der EU-Chemikalienpolitik (Tabelle 1). Weitere Stoffindikatoren sind in der Entwicklung: Für die Bewertung der stofflichen Belastung von Ökosystemen (State) soll ein - noch unbestimmter - ,mariner Bioindikator" aus den Meeresschutzstrategien übernommen werden (Jenseit et al. 2005, S. 45 ff.). Für terrestrische Ökosysteme ist die Indikatorendiskussion noch zu führen.

Im Rahmen der Wirkungsermittlung der Gemeinsamen Agrarpolitik (GAP) der EU auf die Umwelt wurden 35 sogenannte IRENA-Indikatoren nach dem DPSIR-Ansatz (EEA 2006) mit Fragestellungen im stofflichen Bereich entwickelt, zu denen neben Nährstoffen auch das Vorhandensein von Pestiziden in Boden und im Grundwasser gehören (vgl. Tabelle 1). Die IRENA-Indikatoren haben sich als sinnvoll für eine europaweite Analyse der Umweltwirkungen der Landwirtschaft erwiesen, sind jedoch nicht ausreichend mit regionalen nationalen Daten untersetzt, um differenzierte nationale oder gar regionale Bewertungen durchzuführen.

2.4 Erfolgskontrolle in Deutschland:

Vollzugsspezifische Umweltbeobachtung

Die Begrenzung von schädlichen Stoffeinträgen in die Umwelt ist Aufgabe vieler Umweltgesetze und mündet in eine Vielzahl - bislang unverknüpfter - Einzelmaßnahmen und 
Tabelle 1 Stoffliche Indikatoren auf EU-Ebene

\section{DPSIR}

Den Verlust der Biodiversität anhalten: SEBI-Indikatoren (EEA 2007)

Pressure Indicator 9: Critical Load exceedance for nitrogen: Exceedance of critical loads for nitrogen deposition indicating risks for biodiversity loss in (semi)-natural ecosystems

Indicator 15: Nutrients in transitional, coastal and marine waters: Indicates trends in, and concentrations of, winter nitrate and phosphate (microgram/1), as well as nitrogen/phosphorous ratio in the seas of Europe

Indicator 16: Freshwater quality:

1. Annual median concentrations in rivers of Biological Oxygen Demand (BOD) and ammonium (NH4);

2. Trends in concentrations of orthophosphate and nitrate in rivers, total phosphorus and nitrate in lakes, and nitrate in groundwater bodies

Indicator 19: Agriculture: nitrogen balance: accounts for all inputs to and outputs from the farm, and therefore includes nitrogen input

\section{Integration der Umwelt in die Landwirtschaftspolitik: IRENA Indikatoren (EEA 2006)}

Driving Forces Indicator 8: Fertilizer Consumption

Indicator 9: Consumption of pesticides

Pressure Indicator 18: Gross nitrogen balance

Indicator 18sub: Atmospheric emissions of Ammonia

Indicator 20: Pesticide soil contamination

State Indicator 30.1: Nitrates in Water

Indicator 30.2: Pesticides in water

Pressure

Erschöpfung natürlicher Ressourcen: Indikatoren für die Verbreitung von toxischen Substanzen (EUROSTAT 1999)

Verbrauch von Pflanzenschutzmitteln

Emissionen persistenter organischer Schadstoffe (POP)

Verbrauch giftiger Chemikalien

Index von Schwermetallemissionen in Gewässer

Index von Schwermetallemissionen in die Luft

Emissionen radioaktiven Materials

Emissionen von Schwermetallen durch Verbraucherprodukte

Produktion chlorierter Verbindungen

Verbrauch giftiger Haushaltschemikalien

Fahrzeugverteilung nach Technologieart

Monitoringpflichten. Die Umweltbeobachtungsprogramme sind in der Mehrzahl vollzugsspezifisch ausgerichtet. Die Beobachtung der Umwelt (Naturhaushalt) und Natur (Biodiversität) erfolgt gemäß den administrativen Zuständigkeiten zersplittert. Schutzgutübergreifend organisiert sind lediglich die Umweltprobenbank des Bundes (UBA 2000) und das Messnetz IMIS (integriertes Mess- und Informationssystem zur Überwachung der Radioaktivität). Das Erkennen komplexer Wirkungszusammenhänge ist allein schon wegen des horizontalen Informationsaustausches zwischen verschiedenen Administrationen aufwändig, wenngleich hier zum Teil Verwaltungsvereinbarungen bestehen. Trotz aller Rechtsvorgaben finden sich Chemikalien in steigenden Konzentrationen in ökologischen Systemen und Nahrungsnetzen.

Es besteht somit ein erhebliches Vollzugsdefizit, das durch fehlende horizontale Kooperation innerhalb der an der Bewertung und Kontrolle gefährlicher Stoffe beteiligten Verwaltung mit verursacht wird. Diese institutionellen Hürden sind typisch für das Spannungsfeld zwischen querschnitts- orientierten Schutzzielen, wie z.B. dem Schutz der Biodiversität und der bestehenden fachlich spezialisierten Behördenstruktur (SRU 2004, Abschn. 13.1.1.). Bei schädlichen Chemikalien kommen als weitere Verantwortungsträger die Stoffanwender dazu, die ihrerseits Anwendungsbestimmungen einhalten müssen, um Risiken zu vermeiden (s. u.).

Exemplarisch soll hier das Spannungsfeld zwischen Stoffprüfung, Stoffmanagement und Umweltbeobachtung für Pflanzenschutzmittel und für Industriechemikalien betrachtet werden. Pflanzenschutzmittel (PSM) werden gezielt in die Umwelt eingebracht. Die Zulassungsprüfung sieht die prospektive Bewertung derjenigen Risiken vor, die bei einer ordnungsgemäßen Anwendung der PSM entstehen. Teil der Zulassung sind Anwendungsbestimmungen, die vor Ort einzuhalten sind. Bei der Zulassung ist der Schutz der Biodiversität insbesondere für Nichtzielorganismen durch die Mitgliedstaaten zu gewährleisten (91/414/EG, Anhang VI). Sowohl in der Gewässerüberwachung der Länder als auch in Forschungsprojekten zeigt sich, dass PSM in erheblichem 
Umfang unsachgemäß angewendet werden. Zum Beispiel erwies eine vom UBA beauftragte, nur intern verfügbare Feldstudie von 2006, dass bei $50 \%$ der Erhebungen die Auflagen zur Ausbringung der PSM nicht eingehalten wurden. Eine Auswertung von 41 Monitoringstudien zeigt, dass in mehr als der Hälfte dieser Studien erhebliche, zum Teil längerfristige Auswirkungen auf Lebensgemeinschaften beobachtet wurden (Hommen 2004). Eine gezielte Maßnahmenstrategie erfordert räumlich differenzierte Belastungsdaten, aber auch die Zusammenarbeit der beteiligten Bundes- und Länderbehörden unter Einbeziehung der Anwender. Derzeit sind die Messnetzdichte der Länderprogramme, die erhobenen Parameter und die daraus generierten Informationen uneinheitlich und lückenhaft und eine länderübergreifende Übersicht über die tatsächliche Belastungslage kann nicht generiert werden (SRU 2008, Abschn. 8.6.5). Ein schutzgutbezogenes Monitoring der Wirkungen auf Nichtzieleffekte fehlt.

Industriechemikalien gelangen über Abluft, Abwasser und industrielle Abfälle sowie über Verbraucherprodukte in die Umwelt. Eine Zulassungspflicht besteht seit 2006 nur für besonders besorgniserregende Stoffe („REACH-VO“, EG 2006/1907, Titel VII, Anhang XIII). Die neue REACHVO schreibt erstmals die Registrierung aller Stoffe mit einer Produktionsmenge von mehr als $1 \mathrm{t}$ pro Jahr bei der europäischen Chemikalienagentur vor. Teil der einzureichenden Unterlagen sind ökosystemare Wirkungsschwellenwerte. Die Verantwortung für eine sichere Verwendung der Stoffe tragen Hersteller, Importeure und Stoffanwender. Sie müssen mindestens die Unterschreitung der Wirkungsschwellenwerte für ihre Anwendungen gewährleisten. Bei Stoffen ohne Wirkschwelle (z.B. PBT-Stoffe) gilt ein generelles Minimierungsgebot. Monitoringdaten fließen fakultativ in die Risikobewertung als Kriterium für vertiefte Prüfung ein. Ein eigenes Monitoring ist aber nur im Zulassungsfall für den Hersteller/Anwender vorgeschrieben. Eine Erfolgskontrolle auf Basis von Umweltdaten erscheint - auch aufgrund der Erfahrungen bei der Anwendung von Pflanzenschutzmitteln - geboten, fand aber bisher keinen Niederschlag in rechtlichen Vorschriften. Auch eine stoffübergreifende Indikatorendiskussion zum Umweltzustand und zu den Wirkungen der Gesamtbelastung fehlt.

Die beiden thematischen Beispiele zeigen, dass die Zahl der Verantwortlichen und damit auch die Konfliktpotenziale zwischen konkurrierenden Zielen tendenziell steigen. Umso dringender erscheint der Aufbau einer Erfolgskontrolle und damit einer integrierten Umweltbeobachtung, die Problemschwerpunkte aufzeigt und ein gezieltes Handeln unterstützt.

\section{Ergebnisse}

Viele Tier- und Pflanzenarten in Deutschland sind durch den Eintrag von Schadstoffen in die Ökosysteme gefährdet.
Dem trägt die nationale Biodiversitätsstrategie Rechnung, indem sie die Reduzierung der Stoffbelastung fordert und diese Forderung mit der Entwicklung von ökosystembezogenen Wirkungsschwellenwerten verbindet.

Für die Analyse von diffusen Stoffeinträgen gibt es eine entwicklungsfähige fachliche Grundlage: Tabelle 2 zeigt im Geschäftsbereich des BMU angesiedelte Beobachtungsprogramme mit Bezug zur Biodiversität sowie ihre Vernetzung (ohne Naturschutz) (Heiß 2009).

Konkrete Empfehlungen zur besseren Verknüpfung und Auswertung der zersplitterten Umweltbeobachtungsdaten liegen ebenfalls vor (UBA 2002). Das UBA hält eine umfassende Dokumentation von beschreibenden Informationen zu den Umweltbeobachtungsprogrammen vor, welche auch im Umweltdatenkatalog des Bundes und der Länder online (UDO) zur Verfügung steht. Eine schutzgutorientierte, bundesweit harmonisierte Umweltbeobachtung harrt aber weiterhin der Umsetzung in die Routineaufgaben. Ein wichtiges Anliegen des stoffbezogenen Umweltmonitorings sollte es sein, chemisch-analytische Bestimmungen (Expositions- und Belastungsmonitoring) stärker als bislang mit biologischen Wirkungsuntersuchungen zu verknüpfen und räumlich zu differenzieren, um eine möglichst umfassende Bewertung des Zustandes der untersuchten Ökosysteme zu erreichen (Rüdel et al. 2008). Ebenso dringlich erscheint die Entwicklung geeigneter State- und Impact-Indikatoren für die Erfolgskontrolle der Chemikalienpolitik. Hierfür sowie für die Verknüpfung von Expositions- und Belastungsmonitoring ist das Moosmonitoring ein geeignetes Beispiel im Bereich der Erfassung der Anreicherung von (öko-)toxikologisch relevanten Schwermetallen und Stickstoff (Kap. 4).

\section{Diskussion}

Stabilität und Resilienz von Ökosystemen sind wichtige Voraussetzungen für deren Biodiversität. Die Erfassung der biologischen Vielfalt und der Stabilitätsbedingungen von Ökosystemen - insbesondere der wirkungsrelevanten Stoffanreicherungen - durch Umweltmonitoring ist daher auch Kernbestandteil der UNECE (United Nations Economic Commission for Europe) (1979).

Deutschland beteiligte sich seit 1990 an den Heavy Metals in Mosses Surveys, die von der UNECE im ICP (Integrated Cooperative Programme) Vegetation auf Grundlage des Genfer Luftreinhalteabkommens (Convention on Long-Range Transboundary Air Pollution, CLRTAP) im Fünfjahresturnus an mindestens 1,5 Standorten pro $1000 \mathrm{~km}^{2}$ europaweit durchgeführt werden (Doyle et al. 1996). Dabei werden die Anreicherungen von Schwermetallen (seit 1990) und Stickstoff (seit 2005) ermittelt. Die aktuellste Auswertung der Metallanreicherung in den Moo- 
Tabelle 2 Bausteine für die Integration von Stoffdaten in die Biodiversitätsstrategie (UBA 2002, S. 222 ff., verändert)

\begin{tabular}{|c|c|c|}
\hline $\begin{array}{l}\text { Federführung } \\
\text { der Programme }\end{array}$ & Programm & Verknüpfungen (Auswahl) \\
\hline \multirow[t]{4}{*}{ Länder } & Bodendauerbeobachtungsprogramme der Länder (BDF) & $\begin{array}{l}\text { ICP Forests, Klimamessnetz DWD, UPB, Moos- } \\
\text { monitoring }\end{array}$ \\
\hline & Oberflächen-/Grundwassermonitoring (LAWA-Messnetz) & IKSR, IKSE, IKSO, IKSM \\
\hline & Luftmessnetze der Bundesländer & Berichtspflichten des Bundes für EU \\
\hline & Forstliches Monitoring & WGE-ICPs, UPB, Moosmonitoring \\
\hline \multirow{3}{*}{$\begin{array}{l}\text { Länder mit Beteiligung } \\
\text { des Bundes }\end{array}$} & Moosmonitoring & ICP-Vegetation, Luftmessnetz \\
\hline & Bund/Länder-Messprogramm für die Meeresumwelt (BLMP) & COMBINE (Ostsee), TMAP (Wattenmeer), JAMP \\
\hline & Arzneimittelmonitoring & $\begin{array}{l}\text { LAWA, Arbeitsgemeinschaft für die Reinhaltung der } \\
\text { Elbe (ARGE Elbe), BDF }\end{array}$ \\
\hline \multirow[t]{4}{*}{ Bund (Umweltbundesamt) } & Bodendauerbeobachtungsprogramm im Hintergrundbereich & UPB, ICP-Forests, Level II \\
\hline & Luftmessnetz des UBA & ICP, DWD-Monitoring \\
\hline & Umweltprobenbank des Bundes (UPB) & $\begin{array}{l}\text { TMAP, UBA Luftmessnetz, Moosmonitoring, Natio- } \\
\text { nalparks, Biosphärenreservate }\end{array}$ \\
\hline & Umwelt-Survey & APUG \\
\hline \multirow[t]{3}{*}{$\begin{array}{l}\text { Beitrag zu internationalen } \\
\text { Programmen }\end{array}$} & $\begin{array}{l}\text { Integrated Cooperative Programme ICP (bis zu } 90 \text { Unter- } \\
\text { programme) }\end{array}$ & $\begin{array}{l}\text { UPB, UBA Luftmessnetz, Nationalparks, BDF, } \\
\text { Moosmonitoring }\end{array}$ \\
\hline & $\begin{array}{l}\text { Bestimmung von ökologischen Belastungsgrenzen (Critical } \\
\text { Loads and Levels) }\end{array}$ & $\begin{array}{l}\text { Auswertung der ICP Daten, ICP Modelling and } \\
\text { Mapping }\end{array}$ \\
\hline & EU-Land Cover (CORINE-Programm), & Beitrag zu GMES \\
\hline
\end{tabular}

Abkürzungen

APUG: Aktionsprogramm Umwelt und Gesundheit

CLRTAP: Convention on Long-Range Transboundary Air Pollution

COMBINE: Cooperative Monitoring in the Baltic Marine Environment

CORINE: Coordinated Information on the European Environment

DWD: Deutscher Wetterdienst

EMEP: European Monitoring and Evaluation Programme

GMES: Global Monitoring for Environment and Security

ICP: Integrated Cooperative Programme der UNECE

IKSR, IKSE, IKSO, IKSM: Internationale Kommissionen zum Schutz des Rheins, der Elbe, der Oder und der Maas

JAMP Joint Assessment and Monitoring Programme (Nordsee)

LAWA: Bund/Länder-Arbeitsgemeinschaft Wasser

TMAP: Trilateral Monitoring and Assessment Programme (Wattenmeer)

UBA: Umweltbundesamt

UPB: Umweltprobenbank

WGE: Working Group on Effects

WGSR: Working Group on Strategies and Review

sen Deutschlands zwischen 1990 und 2005 legten Schröder et al. (2009) vor, die europäischen Ergebnisse präsentierten Harmens et al. (2008, 2009). In einer bislang unveröffentlichten Auswertung anlässlich des COST Workshops Nitrogen and Natura 2000 vom 18. bis 20. Mai 2009 in Brüssel wurde aufgezeigt, dass das Messnetz des Moosmonitorings in der Lage ist, die Schwermetall- und Stickstoffexposition für die 3492 FFH-Gebiete in Deutschland (Sites of Community Importance, SCI) zu quantifizieren. Demnach lässt sich ein statistisch signifikanter Rückgang der Schwermetallanreicherungen zwischen 1990 und 2000 nachweisen, zwischen 2000 und 2005 ein statistisch signifikanter Anstieg. Sowohl die Metall- als auch die Stickstoffexposition konnte naturräumlich und nach Bundesländern differenziert ausgewertet werden.

\section{Schlussfolgerungen}

Neben den fachlichen Voraussetzungen hängt der Erfolg der Biodiversitätsstrategie entscheidend davon ab, wie konkret sie eine, an den Schutzgütern ausgerichtete, Erfolgskontrolle implementieren kann. Hier gibt es fachbereichs- und auch länderübergreifende Ansätze, wie zum Beispiel den Umweltbericht für das Biosphärenreservat Rhön, die als Modell dienen können. Das Moosmonitoring ist ein medienübergreifendes Programm, das über drei räumliche und administrative Ebenen - Region (z.B. Bundesland oder Naturraum), Staat (z. B. Deutschland) und Kontinent (Europa) - Daten bereitstellt.

Entscheidend ist, dass vorhandene Messprogramme entsprechend ihrer fachlichen Komplementarität miteinander 
verknüpft und nach Möglichkeit medienübergreifend ausgewertet werden. Die technischen Möglichkeiten hierfür sind vorhanden, man sollte sie nutzen.

\section{Empfehlungen und Ausblick}

Kooperationsvereinbarungen, Verfahren zur Lösung von Zielkonflikten und ein anspruchsvolles Daten- und Informationsmanagement stehen erst am Anfang. Ihnen gilt es gezielter Aufmerksamkeit zu widmen als bisher. Der Bedarf an Daten und Indikatoren für die Fortschrittskontrolle komplexer Strategien stellt eine Chance dar, die festgefahrene Reform für eine integrierte Umweltbeobachtung neu zu beleben. Um eine zielgerichtete Verbesserung der Erfolgskontrolle und der Datengrundlage für Indikatoren zu erreichen, ist eine Initiative zur Umsetzung und Weiterführung der vorhandenen Konzepte zur integrierten Umweltbeobachtung notwendig. Auch wenn das Jahr 2015 als Ziel für die Festlegung von Wirkungsschwellenwerten für Schadstoffe in der nationalen Biodiversitätsstrategie noch weit in der Zukunft zu liegen scheint, zeigen die Umsetzungsschwierigkeiten der vergangenen 20 Jahre, dass man nicht zu früh beginnen kann.

\section{Literatur}

BMU (2000) Umweltbeobachtung - Stand und Entwicklungsmöglichkeiten. Vorlage zur 25. Amtschefkonferenz am 23./24. März 2000 in Berlin

BMU (2006) Schadstoffregister PRTR. http://www.bmu.de/luftreinhaltung/downloads/doc/36906.php (letzter Zugriff am 9. Februar 2009)

BMU (Bundesministerium für Umwelt, Naturschutz und Reaktorsicherheit) (2007) Nationale Strategie zur biologischen Vielfalt. Vom Bundeskabinett am 7.11.07 beschlossen. BMU, Berlin. www.biodiv.org (letzter Zugriff am 14. August 2009)

Broecker F, Schmidt G, Schröder W (2007) Medienübergreifende Untersuchung zur Versauerung in Baden-Württemberg. In: Wichmann HE, Schlipköter HW, Füllgraf G (Hrsg) Handbuch der Umweltmedizin. Ecomed, Landsberg am Lech, Kap. IV-7.1, 17-19

Doyle U, Herpin U, Markert B, Siewers U, Lieth H, Bau H (1996) Monitoring der Schwermetallbelastung in der Bundesrepublik Deutschland mit Hilfe von Moosanalysen am Beispiel des Konzentrationsmusters von Blei. Verh Ges Ökologie 26:17-23

Doyle U, von Haaren C, Ott K, Leinweber T, Bartolomäus C (2005) Noch fünf Jahre bis 2010 - eine Biodiversitätsstrategie für Deutschland. Natur Landsch 80(8):349-354

Dröschmeister R, Benzler A, Berhorn F, Doerpinghaus A, Eichen C, Ftitsche B, Graef F, Neukirchen M, Sukopp U, Weddeling K, Züghart, W (2006) Naturschutzmonitoring: Potenziale und Perspektiven. Natur Landsch 81(12):578-584

Europäische Kommission (2008) EU Biodiversity Action Plan Report 2008. A mid-term assessment of implementing the EC Biodiversity action plan. SEBI 2010 biodiversity indicators. SEC(2008) ddd. Europäische Kommission, Brüssel

EEA (2006) Integration of environment into EU agriculture policy the IRENA indicator-based assessment report. Copenhagen: EEA. EEA Report 2/2006
EEA (European Environment Agency) (2007) Halting the loss of biodiversity by 2010: proposal for a first set of indicators to monitor progress in Europe. Copenhagen: EEA. EEA Technical report $11 / 2007$

Ellenberg H, Fränzle O, Müller P (1978) Ökosystemforschung im Hinblick auf Umwelt- und Entwicklungsplanung. Umweltforschungsplan des Bundesministers des Innern, Ökologie-Forschungsbericht 78-101 04 005. Bonn, $144 \mathrm{~S}$

EUROSTAT (1999) Towards Environmental Pressure Indices. http:// esl.jrc.it/envind/(Indicator menu: http://esl.jrc.it/envind/hm_me de.htm) (letzter Zugriff am 9. Februar 2009)

Ferretti M (2001) Ecosystem monitoring. From the integration between measurements to the integration between networks. Publicazione del Corso di Cultura in Ecologia, Università degli studi di Padova, S 3-54

Genßler L, Rademacher J, Rammert U (2001) Arbeitskreis Bioindikation und Wirkungsermittlung der Landesanstalten und -ämter. Konzeption der künftigen Aufgabenbereiche. Umweltwiss Schadst Forsch 13:1-4

Harmens H, Norris D (2008) Spatial and temporal trends in heavy metal accumulation in mosses in Europe (1990-2005). Programme Coordination Centre for the ICP Vegetation, Centre for Ecology and Hydrology, Environment Centre Wales, Bangor, UK

Harmens H, Norris D, Cooper D, Hall J (2009) Spatial trends in nitrogen concentrations in mosses across Europe in 2005/2006. Programme Coordination Centre for the ICP Vegetation, Centre for Ecology and Hydrology, Environment Centre Wales, Bangor, UK

Heiß C (2009) Was kann die stoffbezogene Umweltbeobachtung für die Biodiversitätsstrategie leisten? Naturschutz und Biologische Vielfalt. Im Druck

Hommen U (2004) Auswertung der wichtigsten in Deutschland durchgeführten Monitoringstudien zu Auswirkungen von Pflanzenschutzmitteln auf Nichtzielorganismen. Bericht des FraunhoferInstitutes für Molekularbiologie und Angewandte Ökologie (IME). http://ubanet/Gruppen/bibliothek/Eingangsspeicher\%2D fuer $\% 2$ DLit $\% 2$ Daus\%2DUFORDATerhebung/Monitoring Auswirkung\%20PSM_NichtzielorganismenVH1010300.pdf (letzter Zugriff am 9. Februar 2009)

Jenseit W, Bunke D, Rheinberger U, Kalberlah F, Akkan Z, Moll S (2005) Research, Development, Statistical and Analytical Work to Develop Appropriate Environmental Indicators Related to Chemicals Report Phase III, im Auftrag von EUROSTAT, Luxemburg

Knetsch G, Rosenkranz D (2003) Umweltbeobachtung - Konzepte und Programme des Bundes. In: Landesamt für Umwelt und Geologie Freistaat Sachsen (Hrsg) Ziele, Strategien, Konzepte des Bundes und ausgewählter Länder. Dresden, S 6-24

Koch H-J, Krohn S (2008) Das Naturschutzrecht im Umweltgesetzbuch. Den Auftrag der Föderalismusreform erfüllen. Forum Umweltgesetzbuch 7. Umweltbundesamt, Dessau

LANA (Länderarbeitsgemeinschaft Naturschutz) (2003) (Ökologische) Umweltbeobachtung des Bundes und der Länder. Vorlage zur 60. Umweltministerkonferenz am 15./16. Mai 2003 in Hamburg

OSPAR (Convention for the Protection of the Marine Environment of the North-East Atlantic) (2007) OSPAR List of Chemicals for Priority Action (update 2007). http://www.ospar.org/documents/dbase/decrecs/agreements/04-12e_List $\% 20$ of $\% 20$ Chemicals $\% 20$ for $\% 20$ Priority\%20action.doc (letzter Zugriff am 9. Februar 2009)

Rüdel H, Bester K, Eisenträger A, Franzaring J, Haarich M, Köhler J, Körner W, Oehlmann J, Paschke A, Ricking M, Schröder W, Schröter-Kermani C, Schultze T, Schwarzbauer J, Theobald N, von der Trenck T, Wagner G, Wiemüller GA (2008) Positionspapier zum stoffbezogenen Umweltmonitoring. Umwelt Forsch Prax 13(3):155-164 
Sächsischer Landtag (2008) Biodiversität im Freistaat Sachsen. Dresden: Sächsisches Staatsministerium für Umwelt und Landwirtschaft. Drucksache 4/11590

Schönthaler K, Andrian-Wehrburg S von (2009) Erster integrierter Umweltbericht für das länderübergreifende Biosphärenreservat Rhön. Natursch Landschaftspl 41(1):5-14

Schröder W, Schmidt G (2003) Medienübergreifende Umweltbeobachtung in Baden-Württemberg. Ergebnisse eines Modellprojekts. In: Landesanstalt für Umweltschutz (Hrsg) Medienübergreifende Umweltbeobachtung. Stand und Perspektiven. Karlsruhe, S 39-60

Schröder W, Pesch R, Matter Y, Göritz A, Dieffenbach-Fries H, Genßler L (2009) Trend der Schwermetall-Bioakkumulation 1990 bis 2005: Qualitätssicherung bei Probenahme, Analytik, geostatistischer Auswertung. Umweltwiss Schadst Forsch (in Begutachtung)

SRU (1991) Allgemeine ökologische Umweltbeobachtung. Sondergutachten. Oktober 1990. Metzler-Poeschel, Stuttgart

SRU (2002) Für eine Stärkung und Neuorientierung des Naturschutzes. Sondergutachten. Metzler-Poeschel, Stuttgart
SRU (2004) Umweltgutachten 2004. Umweltpolitische Handlungsfähigkeit sichern. Nomos, Baden-Baden

SRU (Sachverständigenrat für Umweltfragen) (2008) Umweltgutachten 2008. Umweltschutz im Zeichen des Klimawandels. Erich Schmidt, Berlin

UBA (Umweltbundesamt) (2000) Umweltprobenbank des Bundes. http://anubis.uba.de/wwwupb/servlet/upb (letzter Zugriff am 9. Februar 2009)

UBA (2002) Endbericht Projekt Umweltbeobachtung. Unveröffentlicht, Berlin

UMK (Umweltministerkonferenz) (2007) Erfahrungsbericht Indikatoren der Bund-Länder-Arbeitsgemeinschaft Nachhaltige Entwicklung - BLAG-NE https://www.umweltministerkonferenz.de/uploads/Endstand_Protokoll_UMK_Fassung_13_44b.pdf (letzter Zugriff am 14. August 2009)

UNECE (United Nations Economic Commission for Europe) (1979) Convention on Long-range Transboundary Air Pollution (LRTAP) http://www.umweltbundesamt.de/umweltbeobachtung/uid/unece/index.htm (letzter Zugriff am 9. Februar 2009) 